$45-50$

\title{
Surgical treatment of multiple spine metastases from gastrinoma
}

Authors Kelli L Crabtree, Karen K Anderson, Neal G Haynes, Paul M Arnold

Institution University of Kansas Medical Center, Kansas City, KS, USA

\section{ABSTRACT}

Study design: Case report.

Clinical question: To report successful surgical therapy for spinal cord compression in a patient with spinal metastases from a pancreatic gastrinoma.

Methods: A 43-year-old man presented three times within 4 years with cervical and upper thoracic spinal cord compression because of metastatic gastrinoma. He had two previous spine metastases to the lower thoracic and lumbar spine, a T11 compressive lesion which required a T9L1 fusion, and an L4 lesion that was treated with chemotherapy and stereotactic radiation. The compression was relieved each time by surgery.

Results: The patient underwent three surgeries in 4 years: (1) debulking and removal of the rib head on the left at T3, and debulking of the tumor at T3 with hemilaminectomy and spinal cord decompression with internal fixation from T1-T5 using posterolateral instrumented fusion and allograft; (2) anterior C7 corpectomy with placement of a cage from C7-T1 with both anterior and posterior fusion of C2C7; and (3) T1-T3 laminectomy, T1-T3 exploration of wound, revision of hardware, T1-T3 removal of spinal tumor, and T3 bilateral transpedicular circumferential decompression. The patient is alive and regained the ability to walk 8 years after initial diagnosis, despite the appearance of spinal metastases 1 year after the diagnosis of liver metastases.

Conclusion: Surgery for spinal cord compression in patients with metastatic neuroendocrine tumors can be effective in relieving radicular pain, weakness and numbness, and while not curative can greatly improve quality of life.

No funding was received for this work, nor are there any conflicts of interest. 


\section{INTRODUCTION}

Pancreatic endocrine tumors (PETs) are relatively rare, having a clinical detection rate of 1:100,000 which comprise $1 \%-2 \%$ of all pancreatic tumors [1, 2]. PETs are considered functional or nonfunctional and may be sporadic or inherited but little is known about their molecular pathogenesis [1-3]. There is currently no formal classification system for PETs. Their clinical presentation varies depending on whether the tumor is functional [1]. It is believed that $20 \%-25 \%$ of gastrinoma is due an inherited disorder termed multiple endocrine neoplasia type 1 (MEN1) [1, 4]. Gastrinomas are gastrin-secreting functional PETs and cause increased acid secretion resulting in peptic ulcer formation, known as the Zollinger-Ellison syndrome (ZES $[1,5]$. There are two major treatment goals for ZES: (1) to control the acid hypersecretion so that the ulcer can heal and (2) to remove the gastrinoma itself. With administration of proton pump inhibitors, acid secretion can be controlled in almost all patients $[6,7]$.

Most PETs are relatively indolent but ultimately malignant $[1,8]$, and surgical removal of a gastrinoma is the only modality that offers the possibility of cure. Debulking surgery is often considered useful in unresectable patients [1], and since many patients with MEN-1 or sporadic gastrinomas have multifocal or microscopic lesions, debulking may be the only surgical option and is considered by some to be controversial $[6,9]$.

Bone metastases occur in $7 \%$ of patients with gastrinomas and only when there are concurrent liver metastases, do bone metastases occur in $31 \%[10,11]$. The axial skeleton is the most frequent site of bone metastases in patients with gastrinomas, although more distal metastases can occur early in up to $15 \%-30 \%$ of patients [10]. We report successful surgical therapy for spinal cord compression performed three times within 4 years in a patient with spinal metastases due to metastatic pancreatic gastrinoma.

\section{CASE REPORT}

In October 2007, a 42-year-old man presented for evaluation of back pain of 4 months' duration (Fig 1). His history included symptomatic treatment in 2001 for diarrhea and abdominal pain. Three years later in 2004 the patient had imaging studies that showed multiple liver lesions and a mass in the pancreas. The gastrin level was elevated at $23,000 \mathrm{pg} / \mathrm{mL}$. The patient was diagnosed with ZES and metastatic gastrinoma. He received three treatments of chemoembolization, octreotide, and high doses of nexium at that facility. One year later, he presented to another facility with back pain, and imaging revealed a tumor

Fig 1 Patient sampling and selection.

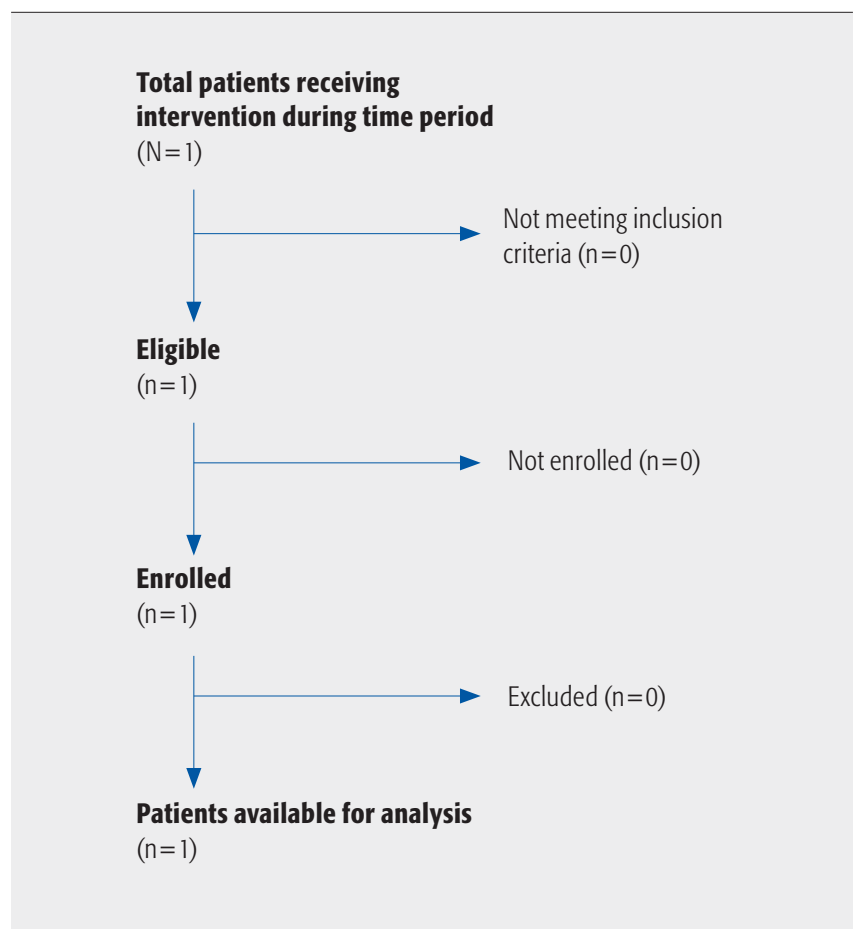

Fig 2 Computed tomographic scan shows tumor destruction of body, pedicle, and lamina of T3 with spinal cord compression.

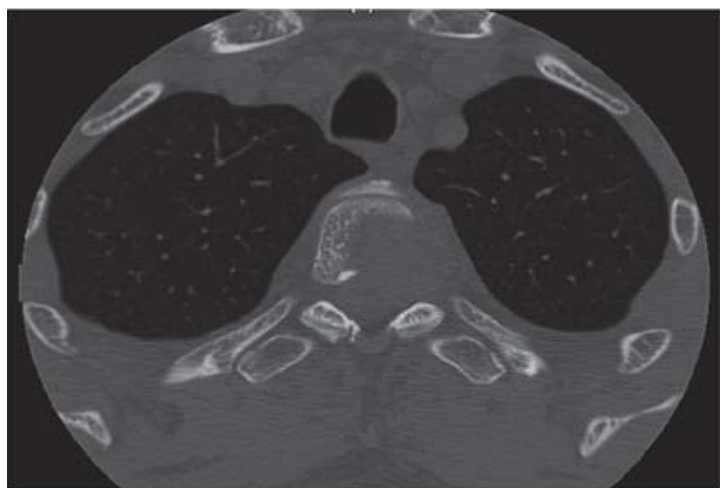


at T11. He underwent resection of the tumor and fusion of T9-L1, followed by radiation. A few months later in October 2005 the patient complained of left hip pain and was found to have another metastasis. He underwent stereotactic radiation therapy to the left hip, L4, and coccyx at that facility in January 2006, and was administered $1000 \mathrm{mg}$ streptozocin and $1500 \mathrm{mg}$ fluorouracil (5-FU) for approximately 6 months. The maintenance therapy of capecitabine [Xeloda®] was not well tolerated and was discontinued.

At the time of his visit in October 2007, computed tomography demonstrated a large metastasis to the left pedicle, vertebral body, and left lamina of T3 causing lateral compression of the spinal cord (Fig 2 ). The patient described intermittent numbness over the left side of his chest that disappeared when he was able to move his arms and mild tenderness to palpation over his upper thoracic spine. He was otherwise neurologically intact with normal motor, sensory, and deep tendon reflexes on detailed neurological examination. He underwent tumor debulking with removal of the rib head on the left with spinal cord decompression, instrumented fixation, and posterolateral fusion with allograft from T1-T5. The patient's symptoms improved. Pathological examination revealed metastatic gastrinoma. Immunohistochemical stains showed the tu-

Fig 3 A pathological specimen shows positive staining for (a)

hematoxylin-eosin x200; (b) synaptophysin x200; (c) chromogranin x200; (d) pan-cytokeratin x400; and (e) gastrin $\times 280$.
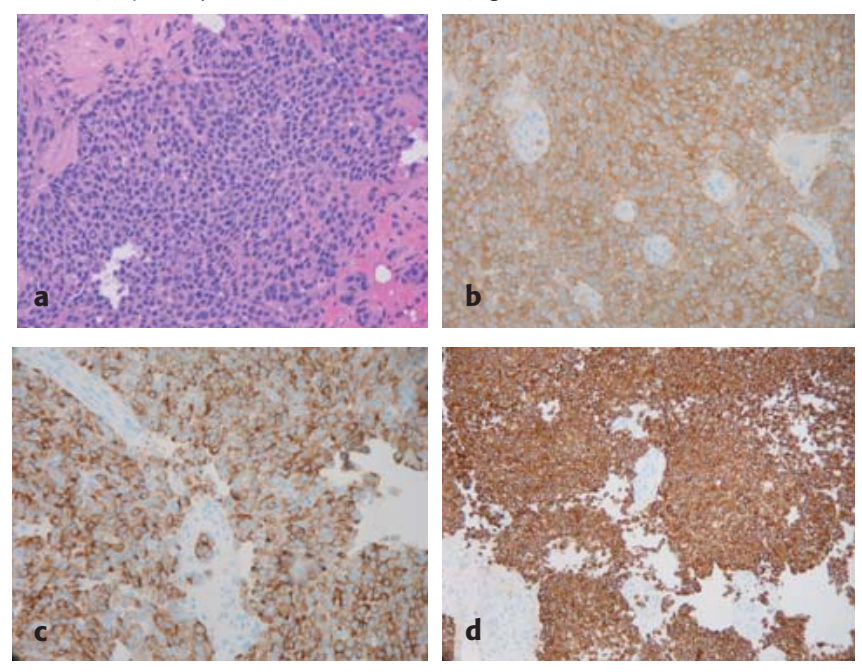

mor cells were positive for synaptophysin, chromogranin, pan-cytokeratin and gastrin, supporting the diagnosis (Fig 3). He was discharged home 3 days postoperatively; his pain was controlled with oral medications, and was instructed to wear the thoracolumbosacral orthosis when out of bed. He then underwent radiation from T2-T4, which was completed end of November 2007. The patient received $4 \mathrm{mg}$ zoledronic acid [Zometa®] intravenously for bone protection every 4 weeks. In January 2008 he underwent SIR-spheres to the hepatic artery via interventional radiology.

Eighteen months later, in May 2009, he again presented to the hospital with left arm numbness and tingling of approximately 3 weeks' duration, as well as onset of numbness and tingling below T4 of 2 days' duration. His symptoms did not improve with dexamethasone therapy [Decadron $®$ ]. He was currently receiving radiation therapy to a mass surrounding C7. On examination he was neurologically intact with the exception of $4 / 5$ strength to left finger extension, and hyperreflexia in the bilateral upper extremities and lower extremities. Magnetic resonance imaging of the C-spine showed a diffuse cervical metastatic lesion centered at the $\mathrm{C} 7$ vertebral body with central canal and left neuroforaminal stenosis (Fig 4). Computed tomography of the T-spine was somewhat limited secondary to previous hardware placement; however, it revealed a metastatic lesion at the T3 level as well as metastasis to several ribs.

The patient underwent a C7 corpectomy with placement of a cage from $\mathrm{C} 6-\mathrm{T} 1$ and posterior fusion of $\mathrm{C} 2-\mathrm{C} 7$. The patient was able to ambulate with walker before discharge.

Fig 4 Magnetic resonance imaging reveals circumferential compression of the spinal cord at C7.

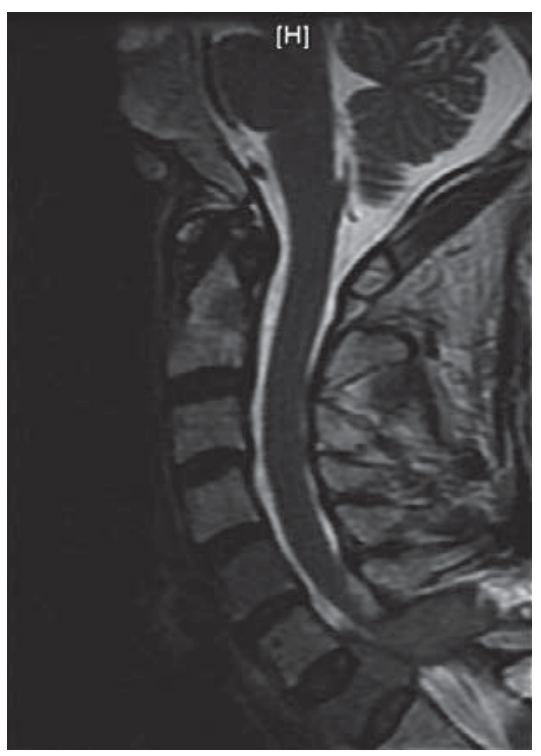


Pathological examination revealed metastatic neuroendocrine carcinoma consistent with his previous diagnosis. Immunohistochemical stains were positive for chromogranin, synaptophysin, CD56, pan-cytokeratin, and focally weakly positive for gastrin.

One week later, the patient reported waking up with weakness in bilateral LEs and an inability to stand or walk. Motor examination demonstrated normal upper extremity strength bilaterally. Strength 3/5 was demonstrated in hip and knee flexion and knee extension bilaterally, and 4/5 in plantar flexion and dorsiflexion and extensor hallucis longus bilaterally. Sensation was intact to light touch, pinprick, and proprioception throughout; although the patient reported altered sensation below level of T4 and in left fingers. Reflexes were $3+$ throughout.

Computed tomographic myelogram showed a complete myelographic block at $\mathrm{T} 3$ with a large destructive mass involving the T3 vertebral body with lateral extension into the posterior pleural space and posterior extension resulting in near complete compression of the spinal cord. Due to the metastatic lesion and spinal cord compression at $\mathrm{T} 3$, the patient underwent T1-T3 laminectomy, revision of hardware, and $\mathrm{T} 3$ bilateral transpedicular and circumferential spinal cord decompression.

Pathological examination again revealed metastatic neuroendocrine carcinoma, infiltrating the bone with fibrous reaction and associated necrosis. Comparison of the $\mathrm{T} 3$ paraspinous mass was made to the recently resected cervical spine mass and both tumors showed identical histological features.

It is now 8 years after initial diagnosis and it has been 12 months since the most recent surgery. He is able to ambulate with assistance, though is experiencing functional decline due to progression of disease.

\section{DISCUSSION}

In general, up to $40 \%$ of patients with cancer will develop skeletal metastases, of which the spine is the most frequent location $[12,13]$. The most commonly affected location is the thoracic spine (up to $70 \%$ of cases), followed by the lumbar and cervical spine [12].

Of all patients who develop spinal metastases, only $5 \%-$ $10 \%$ develop an epidural spinal cord compression, of which only $10 \%-20 \%$ will become symptomatic $[12,14]$. The treatment of spinal metastases remains primarily palliative
[12]. The goals of surgery are to prevent or reverse neurological deterioration and to relieve pain $[12,15]$. Indications for surgery include intractable pain, radiotherapy failure, deformity, spinal instability, and neural compression secondary to retropulsed bone or tumor mass [12, 16, 17]. Determination of surgical objective, timing, and technique must consider mortality and morbidity risks, the location and extent of metastatic disease, the rate of neurological decline, the patient's ability to tolerate the procedure and their overall estimated life expectancy [10, 12].

Pancreatic endocrine tumors (PETs) can vary in regards to their malignant potential, tumor location [1], and rate of progression. Excluding insulinomas, metastases develop in $50 \%-90 \%$ of PET cases and most commonly involve the lymph nodes and liver but can also spread to distant sites, such as the spine and pelvis [1, 2, 18, 19]. In gastrinomas specifically, $75 \%$ of cases arise sporadically while the other $25 \%$ of tumors are associated with MEN-1[6]. Gastrinomas are typically found in the pancreas or duodenum, although they are rarely found in extrapancreatic sites, like the heart and ovary [6]. Up to $30 \%$ of pancreatic gastrinomas and $10 \%$ of duodenal gastrinomas have already metastasized at the time of initial diagnosis [6, 20]. Even in malignant cases, gastrinomas typically follow a relatively indolent disease course [1].

In the past the main cause of morbidity and mortality in ZES was related to complications of fulminant peptic ulcer disease; total gastrectomy was the only treatment effective at preventing acid hypersecretion [21]. With the recent advancements in acid suppression therapy, like $\mathrm{H} 2$ antagonists and proton pump inhibitors, this is no longer the case [22]. Metastatic tumor spread is now the most common cause of morbidity and mortality in patients with gastrinoma [23]. Currently, complete surgical tumor resection is the only potential means of curing patients with PETs [1]. Even when complete resection is not possible, debulking surgery can help alleviate symptoms in many patients [1]. While surgery is promising, it is not likely to be successful in patients with diffuse metastatic disease, or in those who are medically unstable or unable to tolerate the procedure [1]. In cases of gastrinoma due to ZES, the only surgical candidates are those patients with the sporadic form [1, 4, 24, 25].

Like other PETs, the liver is the major metastatic site for gastrinomas $[6,11,20]$. The second most common location is bone, of which the spine and sacrum are the most likely sites, especially later in the disease course. Bone metastases occur in 7\% of all patients with gastrinoma, and occur in $31 \%$ of those same patients with existing liver metastases $[10,11,23,26]$. Thus, it is important that patients with PET with metastases to the liver are carefully 
evaluated for bone metastases since detection of these lesions will change the patients' overall management and affect their prognosis [10]. Somatostatin receptor scintigraphy and magnetic resonance imaging are the best tests to detect these lesions, although the former is preferred because of its ability to image the entire body and detect the extra-axial lesions that can potentially arise $[1,6,27]$.

Identifying bone metastases in patients with ZES is critical to ensure they receive the appropriate treatment plan. One study [10] showed that the detection of bone metastases in patients with gastrinoma lead to an alteration in the chemotherapy regimen or initiation of chemotherapy in $50 \%$ of patients, or in $62 \%$ of patients, an initiation of radiation therapy or other therapies in an attempt to address their bone pain. Changes in other antitumor treatments may also occur in patients with both metastases and slow-growing tumors; for example, the use of interferon or somatostatin analogs is commonly delayed until aggressive tumor growth is suspected [10]. In addition, once patients with ZES are identified as having bone metastases, they are no longer a candidate for other common therapeutic options, such as curative tumor resection, cytoreductive surgery, liver transplantation, or chemoembolization [10]. However, few effective therapies currently exist for patients with ZES with bony metastases or inoperable tumors, but current trials assessing novel treatments, like peptide receptor radiation therapy with a radiolabeled somatostatin analogue are promising $[6,28]$.

\section{CONCLUSION}

Surgical management remains the strongest option in patients with spine metastases and neurological compromise [29]. While spinal decompressive surgery in patients with metastatic neuroendocrine tumors is not curative, it can be effective in relieving radicular pain, weakness and numbness; thus greatly improving quality of life.

\section{REFERENCES}

1. Metz DC, Jensen RT (2008) Gastrointestinal neuroendocrine tumors: pancreatic endocrine tumors. Gastroenterology; 135(5):1469-1492.

2. Oberg K, Eriksson B (2005) Endocrine tumours of the pancreas. Best Pract Res Clin Gastroenterol; 19(5):753-781.

3. Duerr EM, Chung DC (2007) Molecular genetics of neuroendocrine tumors. Best Pract Res Clin Endocrinol Metab; 21(1):1-14.

4. Jensen RT, Berna MJ, Bingham DB, et al (2008) Inherited pancreatic endocrine tumor syndromes: advances in molecular pathogenesis, diagnosis, management, and controversies. Cancer; 113(7 Suppl):1807-1843.

5. Jensen RT, Niederle B, Mitry E, et al (2006) Gastrinoma (duodenal and pancreatic). Neuroendocrinology; 84(3):173-182.

6. Nishio K, Nishio A, Nishikawa T, et al (2007) Recurrent gastrinoma in the mesentery 19 years after primary resection. Dig Dis Sci; 52(11):3104-3108.

7. Hirschowitz BI, Simmons J, Mohnen J (2005) Clinical outcome using lansoprazole in acid hypersecretors with and without Zollinger-Ellison syndrome: a 13-year prospective study. Clin Gastroenterol Hepatol; 3(1):39-48.

8. Gibril F, Venzon DJ, Ojeaburu JV, et al (2001) Prospective study of the natural history of gastrinoma in patients with MEN1: definition of an aggressive and a nonaggressive form. J Clin Endocrinol Metab; 86(11):5282-5293.

9. Jensen RT (1998) Management of the Zollinger-Ellison syndrome in patients with multiple endocrine neoplasia type 1. J Intern Med; 243(6):477-488.

10. Gibril F, Doppman JL, Reynolds JC, et al (1998) Bone metastases in patients with gastrinomas: a prospective study of bone scanning, somatostatin receptor scanning, and magnetic resonance image in their detection, frequency, location, and effect of their detection on management. J Clin Oncol; 16(3):1040-1053.

11. Gibril F, Jensen RT (2005) Advances in evaluation and management of gastrinoma in patients with Zollinger-Ellison syndrome. Curr Gastroenterol Rep; 7(2):114-121.

12. Singh K, Samartzis D, Vaccaro AR, et al (2006) Current concepts in the management of metastatic spinal disease: the role of minimally-invasive approaches. J Bone Joint Surg $B r ; 88(4): 434-442$.

13. Sze WM, Shelley MD, Held I, et al (2003) Palliation of metastatic bone pain: single fraction versus multifraction radiotherapy: a systematic review of randomised trials. Clin Oncol ( $R$ Coll Radiol); 15(6):345-352.

14. Gerszten PC, Welch WC (2000) Current surgical management of metastatic spinal disease. Oncology (Williston Park); 14(7):1013-1024. 
15. Ryken TC, Eichholz KM, Gerszten PC, et al (2003) Evidence-based review of the surgical management of vertebral column metastatic disease. Neurosurg Focus; 15(5):E11.

16. Klimo P Jr, Kestle JR, Schmidt MH (2004) Clinical trials and evidence-based medicine for metastatic spine disease. Neurosurg Clin NAm; 15(4):549-564.

17. Maranzano E, Trippa F, Chirico L, et al (2003) Management of metastatic spinal cord compression. Tumori; 89(5):469-475.

18. de Herder W, O'Toole D, Rindi G, et al (eds) (2006) ENETS Consensus guidelines for the management of patients with digestive neuroendocrine tumors. Part 1-stomach, duodenum and pancreas. Neuroendocrinology; 84(3):151-216.

19. Klöppel G (2007) Tumour biology and histopathology of neuroendocrine tumours. Best Pract Res Clin Endocrinol Metab; 21(1):15-31.

20. Power N, Reznek RH (2002) Imaging pancreatic islet cell tumours. Imaging; 14(2):147-159.

21. Meko JB, Norton JA (1995) Management of patients with Zollinger-Ellison syndrome. Annu Rev Med; 46:395-411.

22. Quatrini M, Castoldi L, Rossi G, et al (2005) A follow-up study of patients with ZollingerEllison syndrome in the period 1966-2002: effects of surgical and medical treatments on long-term survival. J Clin Gastroenterol; 39(5):376-380.

23. Yu F, Venzon DJ, Serrano J, et al (1999) Prospective study of the clinical course, prognostic factors, causes of death, and survival in patients with long-standing Zollinger-Ellison syndrome. J Clin Oncol; 17(2):615-630.

24. Akerström G, Hellman P (2007) Surgery on neuroendocrine tumours. Best Pract Res Clin Endocrinol Metab; 21(1):87-109.

25. Norton JA, Alexander HR, Fraker DL, et al (2001) Comparison of surgical results in patients with advanced and limited disease with multiple endocrine neoplasia type 1 and Zollinger-Ellison syndrome. Ann Surg; 234(4):495-505.

26. Lebtahi R, Cadiot G, Delahaye N, et al (1999) Detection of bone metastases in patients with endocrine gastroenteropancreatic tumors: bone scintigraphy compared with somatostatin receptor scintigraphy. J Nucl Med; 40(10):1602-1608.

27. Virgolini I, Traub-Weidinger T, Decristoforo C (2005) Nuclear medicine in the detection and management of pancreatic islet-cell tumours. Best Pract Res Clin Endocrinol Metab; 19(2):213-227.

28. Forrer F, Waldherr C, Maecke HR, et al (2006) Targeted radionuclide therapy with 90Y-DOTATOC in patients with neuroendocrine tumors. Anticancer Res; 26(1B):703-707.

\section{COMMENTARY}

Author Jean-Paul Wolinsky

Institution Department of Neurosurgery, The Johns Hopkins Hospital, Baltimore, MD, USA

Crabtree et al present the case of a 43-year-old man with metastatic gastrinoma and the surgical treatment that he underwent for spinal disease. The authors provide a review of the natural history and treatment options for gastrinoma. This case report illustrates the indolent nature of the disease and the need to be vigilant in treating this patient population for epidural involvement and spinal cord compression. Unlike patients with metastatic tumors, the life expectancy of those with metastatic gastrinoma can be long; therefore treatment has to be individualized.

The patient underwent four different spinal operations on different sites of spinal cord compression and neurological dysfunction. In other disease states, this might be considered ultraaggressive but as the authors describe this strategy has allowed their patient to be alive and functional for 8 years since his diagnosis and 1 year since his last surgical decompression. As is emphasized in this article, and also described in other papers, surgical treatment for metastatic spinal cord compression can result in a beneficial outcome in quality of life and preservation of neurological function [1, 2].

1. Metz DC, Jensen RT (2008) Gastrointestinal neuroendocrine tumors: pancreatic endocrine tumors. Gastroenterology; 135(5):1469-1492.

2. Patchell RA, Tibbs PA, Regine WF, et al (2005) Direct decompressive surgical resection in the treatment of spinal cord compression caused by metastatic cancer: a randomized trial. Lancet; 366(9486): 643-648. 